OR Spectrum manuscript No.

(will be inserted by the editor)

\title{
The structure of the distributions of cash flows and discount rates in multiperiod valuation problems
}

\author{
Jörg Laitenberger ${ }^{1}$ and Andreas Löffler ${ }^{2 a}$ \\ 1 Wirtschaftswissenschaftliche Fakultät, Universität Hannover, Königsworther Platz 1, D-30167 Hannover, Germany, e-mail: \\ JL@wacc. de \\ ${ }^{2}$ Wirtschaftswissenschaftliche Fakultät, Universität Hannover, Königsworther Platz 1, D-30167 Hannover, Germany, e-mail: \\ AL@wacc.de.
}

Received: date / Revised version: date

Abstract. Abstract is missing.

PACS. XX.XX.XX No PACS code given

n capital budgeting problems future cash flows are discounted using the expected one-period returns of the investment. In this paper we relate this approach to the assumption that markets are free of arbitrage. Our goal is to uncover implicit assumptions on the set of cash flow distributions that are suitable for the capital budgeting method.

Our results are twofold. First we obtain that for deterministic cost of capital the set of admissible cash flow distributions is large in the sense that no particular structure of the evolution of the distributions is implied. We give stylized examples that demonstrate that even strong assumptions on the return distributions do not restrain the shape of the cash flow distributions. This shows that Fama's assertion that the distributions of one-period single returns become more and more skewed cannot be generalized to multi-period budgeting problems.

Secondly, in a subsequent analysis we characterize the cash flow distributions under the additional assumption of a deterministic dividend yield. In this case a linear relationship between returns and cash flows obtains.

Key words. cost of capital, capital budgeting JEL-class.: G 31, D46

\section{Introduction}

According to standard textbook advice, asset valuation is done by discounting the expected future cash flows of

Send offprint requests to: Andreas Löffler

a Corresponding author. The authors thank the Verein zur Förderung der Zusammenarbeit von Lehre und Praxis am Finanzplatz Hannover e. $V$. for financial support. an investment with an appropriate risk-adjusted discount rate. The expectation is taken with regards to the subjective probability of the investor, and the risk adjusted discount rate is given by the expected rate of return of the investment. On the other hand it is well known that in arbitrage free markets the value of a claim is given by the sum of its expected cash flows discounted at the riskless interest rate, where the expectation is taken with respect to the so-called risk neutral measure that is usually different from the subjective probability measure of the investor. In this note we investigate the relationship between the textbook approach and the assumption that markets are free of arbitrage. Specifically, our goal is to uncover implicit assumptions on the set of cash flow distributions on the one hand and the set of discount rates on the other hand that are suitable to compute the net present value of an investment project.

If the discount rate is derived from an equilibrium model as the CAPM the above problem reduces to the question under what assumptions a myopic valuation principle can be applied. This problem was considered by Fama (see [5]), Myers and Turnbull (see [10]), Sick (see [12]), Black (see [3]) and Franke (see [7]). In the context of the CAPM the probability distributions of the one-period returns have to be normal. The implications on the shape of the cash flow distributions are less well known.

Fama investigated the case of a single future cash flow some periods ahead. Later he pointed out that in this case the distribution of cash flows tend to become more and more skewed when the distributions of the one-period single returns are roughly symmetric (see [6].) Myers and Turnbull assume a specific pattern of expectation formation that implies certain linearities in the distributions of future cash flows. They show that in this context a constant value for the risk adjusted discount rate is only obtained when the cash flows follow a pure random walk 
process. Sick investigated additive or multiplicative cash flow processes. Black assumed that both the cash flows of the project and the cash flows of the market portfolio are joint normal.

In this note we take a more general view. We ask under the most general conditions of arbitrage-free markets with rational expectations, what is the acceptable uncertainty in the distribution of future cash flows that allows the application of the net present value method. Specifically we tackle two problems. On the one hand we prove that assumptions on the shape of return distributions alone do not restrict the set of admissible cash flow distributions when no restrictions are imposed on the shape of the distributions of the future firm values and vice versa. Therefore the result of Fama that "distributions of payoffs more than one period ahead are skewed right" cannot be generalized to multiperiod budgeting problems. Our analysis includes the results of equilibrium models as special cases, since these restrict only the shape of the return distributions.

On the other hand we discuss two alternative assumptions on the evolution of cash flows in the hope to obtain testable properties of the return distributions, namely the assumption of weak auto-regressive cash flows and the assumption of a deterministic ratio of cash flows to firm value. It turns out that neither condition is sufficient to obtain the required property. Only both assumptions together lead to strong properties for the returns and can guarantee the cost of capital in capital budgeting to be well defined. Our results are closely related to recent work of Ang and Liu (see [1]). They characterize the joint dynamics of stock prices, dividends and expected returns in a continuous time framework. Their focus is on the predictability of stock returns in empirical analysis, while we are interested in consistent definitions of the valuation process.

The next section presents two examples of a firm where the cash flows have an arbitrary stochastic structure in any future time but cost of capital are constant and the market is free of arbitrage. The second example shows that even the assumption of a positive risk premium is compatible with a riskless firm value. Sufficient conditions on the evolution of cash flows for the cost of capital used in capital budgeting to be well defined are given in the following section. The last section closes the paper. Proofs are given in the appendix.

\section{Cost of Capital: Definition and Examples}

Let $(\Omega, \mathcal{F}, P)$ be a probability space, the information at time $t$ is given by the filtration $\mathcal{F}_{t}$ (for an introduction to conditional expectations and the concept of filtration see [13]). The world ends in $T$, where $T=\infty$ is possible. A firm realizes uncertain cash flows $\widetilde{C F}_{t}$ that are $\mathcal{F}_{t}$-measurable. The value of the firm at time $t$ is denoted by $\widetilde{V}_{t}$. The riskless interest rate is for simplicity time independent and $r_{f}$. If the market is free of arbitrage there is a risk neutral probability measure $Q$ such that the following is true (for a proof see for example $\underline{[2]}$ ):

$$
\widetilde{V}_{t}=\frac{\mathrm{E}_{Q}\left[\widetilde{V}_{t+1}+\widetilde{C F}_{t+1} \mid \mathcal{F}_{t}\right]}{1+r_{f}}
$$

where $\mathrm{E}_{Q}\left[\cdot \mid \mathcal{F}_{t}\right]$ is the conditional expectation under the risk neutral probability measure. For a multiperiod valuation problem, this implies for every time period $t$ :

$$
\widetilde{V}_{t}=\sum_{u=t+1}^{T} \frac{\mathrm{E}_{Q}\left[\widetilde{C F}_{u} \mid \mathcal{F}_{t}\right]}{\left(1+r_{f}\right)^{u-t}}
$$

In the case $T=\infty$ equation (2) follows under the additional assumption of transversality.

Our purpose is to clarify the relationship between arbitrage free markets and capital budgeting. To this end we now define the rate of return: At any future time $t$ the rate of return one period ahead from holding a share of the company is

$$
\tilde{r}_{t}:=\frac{\widetilde{V}_{t}+\widetilde{C F}_{t}}{\widetilde{V}_{t-1}}-1
$$

In a world with uncertainty $\tilde{r}_{t}$ will be a random variable. The expectation of the rate of return $\tilde{r}_{t}$ with respect to the information $\mathcal{F}_{t-1}$ will be denoted as cost of capital of the investment at time $t$. The expectation is taken according to the subjective probability measure of the investor. Therefore the cost of capital are the conditional expectations of the one period returns:

$$
\widetilde{k}_{t}=\mathrm{E}\left[\tilde{r}_{t} \mid \mathcal{F}_{t-1}\right]
$$

Let us now turn to the capital budgeting problem. From (3) and (4) it immediately follows that

$$
\widetilde{V}_{t-1}=\frac{\mathrm{E}\left[\widetilde{V}_{t}+\widetilde{C F}_{t} \mid \mathcal{F}_{t-1}\right]}{1+\widetilde{k}_{t}}
$$

As long as $\widetilde{k}_{t}$ is a random variable it is impossible to compute the expectation $\mathrm{E}\left[\widetilde{V}_{t-1} \mid \mathcal{F}_{\tau}\right]$ for $\tau \leq t-2$, therefore we assume that the cost of capital are deterministic. Only in this case (3) implies

$$
\widetilde{V}_{t-1}=\frac{\mathrm{E}\left[\widetilde{C F}_{t} \mid \mathcal{F}_{t-1}\right]}{1+k_{t}}+\frac{\mathrm{E}\left[\widetilde{V}_{t+1}+\widetilde{C F}_{t+1} \mid \mathcal{F}_{t-1}\right]}{\left(1+k_{t+1}\right)\left(1+k_{t}\right)}
$$

and, going back to the date $t$, the well-known present value formula

$$
\widetilde{V}_{t}=\sum_{u=t+1}^{T} \frac{\mathrm{E}\left[\widetilde{C F}_{u} \mid \mathcal{F}_{t}\right]}{\prod_{s=t+1}^{u}\left(1+k_{s}\right)} .
$$

Observe that with the last equation and equation (2) we have two valuation formulas at hand for the same valuation problem, one using the risk-neutral probability $Q$ and the riskless rate of return $r_{f}$ and one using the subjective probability measure and the cost of capital. 
Notice that for deterministic cost of capital the oneperiod returns will be necessarily serially uncorrelated. For $s>t$ :

$$
\begin{aligned}
\operatorname{Cov}\left[\tilde{r}_{s}, \tilde{r}_{t}\right] & =\mathrm{E}\left[\tilde{r}_{s} \tilde{r}_{t}\right]-\mathrm{E}\left[\tilde{r}_{s}\right] \mathrm{E}\left[\tilde{r}_{t}\right]=\mathrm{E}\left[\tilde{r}_{t} \mathrm{E}\left[\tilde{r}_{s} \mid \mathcal{F}_{t}\right]\right]-k_{s} k_{t} \\
& =\mathrm{E}\left[\tilde{r}_{t} k_{s}\right]-k_{s} k_{t}=0 .
\end{aligned}
$$

This is a priori not restraining much the distribution of $\widetilde{C F}_{t}$ and $\widetilde{V}_{t}$. We expect that in valuation problems with multiple cash flow realizations, the distributions of the cash flows may be interrelated in such a way as to allow the construction of almost any sequence of future cash flows or alternatively any sequence of future firm values that imply rates of return as described by (3) as we will show now. This is to be contrasted with the case of a single cash flow realization at the end of time studied by [6], in which all distributions of firm values are derived backwards from the distribution of the cash flows in the last period. If market conditions allow for estimating the one period returns according to the CAPM, this restricts the set of acceptable return distributions $\tilde{r}_{t}$ to be normal and independent, but does not change anything to the indeterminacy of the cash flow distribution. In Fama's analysis the distribution of the future expected cash flows obtains as a multiplicative stochastic process driven by the law of Bayesian expectation formation. In our setup the cash flow distribution of a single period obtains in exactly the same manner, but with multiple cash flow realizations this is not related to the structure of the distributions of the returns of the investment.

The distributions of the cash flows $\widetilde{C F}_{t}$ are related to the firm values $\widetilde{V}_{t}$ and the returns $\tilde{r}_{t}$ by equation (3):

$$
\widetilde{C F}_{t}=\widetilde{V}_{t-1}\left(1+\tilde{r}_{t}\right)-\widetilde{V}_{t}
$$

Consider for instance a sequence of cash flow realizations $\widetilde{C F}_{t}$ and a sequence of related firm values $\widetilde{V}_{t}$ and denote the ratio of cash flows to the firm value by

$$
\widetilde{d}_{t}:=\frac{\widetilde{C F}_{t}}{\widetilde{V}_{t}}
$$

By the definition of the one-period returns in (3) and the cash flow to firm value ratio we have

$$
\widetilde{C F}_{t}=\frac{\widetilde{d}_{t-1}^{-1}}{1+\widetilde{d}_{t}^{-1}}\left(1+\tilde{r}_{t}\right) \widetilde{C F}_{t-1} .
$$

Assuming iid lognormal returns $1+\tilde{r}_{t}$, uncorrelated to all other entries, and the ratios $\widetilde{d}_{t}$ to be such that

$$
\tilde{A}_{t}=\frac{\widetilde{d}_{t-1}^{-1}}{1+\widetilde{d}_{t}^{-1}}
$$

are lognormal for all $t$ and such that

$\operatorname{Cov}\left[\ln \left(\tilde{A}_{t}\right), \ln \left(\widetilde{C F}_{t}\right)\right]=-\frac{1}{2}\left(\operatorname{Var}\left[\ln \left(\tilde{A}_{t}\right)\right]+\operatorname{Var}\left[\ln \left(1+\tilde{r}_{t}\right]\right)\right.$, we have

$$
\begin{aligned}
\operatorname{Var}\left[\ln \left(\widetilde{C F}_{t}\right)\right] & =\operatorname{Var}\left[\ln \left(\tilde{A}_{t}\right)\right]+\operatorname{Var}\left[\ln \left(1+\tilde{r}_{t}\right)\right] \\
& +\operatorname{Var}[\ln (\widetilde{C F} t-1)]+2 \operatorname{Cov}\left[\ln \left(\tilde{A}_{t}\right), \ln (\widetilde{C F} t-1)\right] \\
& =\operatorname{Var}[\ln (\widetilde{C F} t-1)]
\end{aligned}
$$

implying a sequence of lognormal cash flow distributions having a growing variance but a constant skewness even for large $t$. Therefore in general the skewness of the distributions of $\widetilde{C F}_{t}$ is not increasing, as has been claimed by Fama for the case of a single cash flow.

In the following example we show that even the assumption of a positive risk premium does not imply a risky firm value. The uncertainty is fully embedded in the cash flows. To this end consider a sequence of iid random variables $\widetilde{C F}_{t}$ for all $t$. In order to prevent arbitrage we only need two arbitrary probability measures $Q, P$ such that

$$
\mathrm{E}_{Q}\left[\widetilde{C F}_{t}\right]=r_{f}, \quad \mathrm{E}\left[\widetilde{C F}_{t}\right]=k \neq r_{f} .
$$

The filtration $\mathcal{F}_{t}$ is implicitly determined by the sequence of random variables $\widetilde{C F}_{1}, \ldots, \widetilde{C F}_{t}$. We set he value of the firm equal to one: for all $t$

$$
\widetilde{V}_{t}:=1 .
$$

Notice that the value of the firm is not a random variable anymore, although we continue to use the tilde. It is straightforward to verify that this model is free of arbitrage: given the definition of the riskless interest rate we have

$$
\frac{\mathrm{E}_{Q}\left[\widetilde{C F}_{t}+\widetilde{V}_{t} \mid \mathcal{F}_{t-1}\right]}{\widetilde{V}_{t-1}}=1+r_{f},
$$

showing our model is free of arbitrage. On the other hand, the cost of capital are given by

$$
\frac{\mathrm{E}\left[\widetilde{C F}_{t}+\widetilde{V}_{t} \mid \mathcal{F}_{t-1}\right]}{\widetilde{V}_{t-1}}=1+k
$$

We arrive at a situation where the cost of capital of our firm are constant although the risk of the cash flows does not change: in every period the cash flows are given by the same random variable with an arbitrary distribution. The risk of the cash flows does not increase. The value of the firm can be evaluated by discounting the expected cash flows using the cost of capital. In this case the value of the firm obtains as

$$
V_{0}=\sum_{t=1}^{\infty} \frac{\mathrm{E}\left[\widetilde{C F}_{t}\right]}{(1+k)^{t}}=1
$$

Observe that the firm value in $t-1$ can also be computed as:

$$
\widetilde{V}_{t-1}=\frac{\mathrm{E}\left[\widetilde{C F}_{t} \mid \mathcal{F}_{t-1}\right]}{1+k^{*}}+\frac{1}{1+r_{f}}
$$

for some real number $k^{*} \neq k$, since the firm value one period ahead is known to be one. Even though there is 
nothing wrong with computing firm values like this, this is not what the present value is meant to be, since in this case $k^{*}$ is not the expected one-period return of the investment.

Notice that in the last example the expectation and even the variance of the future cash flows remain constant. Hence, a positive risk premium does not imply increasing risk of the future cash flows measured in terms of variance.

\section{Cash flows with a deterministic ratio of cash flows to firm value}

Consider again the ratio of cash flows to firm values

$$
\widetilde{d}_{t}:=\frac{\widetilde{C F}_{t}}{\widetilde{V}_{t}}
$$

It is a frequently used assumption in multiperiod valuation problems to assume that this ratio is deterministic: This is for instance the underlying assumption in Merton's proportional dividend-yield option pricing model (see [9]). Geske is more general and used an independent dividend yield in his model (see [8]).

If both the dividend ratio and the cost of capital are deterministic then the cash flows have to satisfy the relation

$$
\forall t \quad \mathrm{E}\left[\widetilde{C F}_{t+1}-\widetilde{C F}_{t} \mid \mathcal{F}_{t}\right]=g_{t+1} \cdot \widetilde{C F_{t}} .
$$

where $g_{t+1}$ is deterministic. Observe that this assumption is equivalent to saying that the future cash flows form a weak auto-regressive process of degree one (the process under consideration is weak auto-regressive, because we allow for time-varying but deterministic $g_{t}$, see for instance [11]). This implies that the cash flows are autocorrelated and that the increments are uncorrelated and can be seen as follows: Define the cash flow increments

$$
\widetilde{\varepsilon}_{t+1}:=\widetilde{C F}_{t+1}-\left(1+g_{t+1}\right) \widetilde{C F}_{t} .
$$

Using (8) these $\varepsilon_{t}$ obviously have expectation zero. The correlation between two increments can be written as

$\operatorname{Cov}\left(\widetilde{\varepsilon}_{t+1}, \widetilde{\varepsilon}_{s+1}\right)=\mathrm{E}\left[\left(\widetilde{C F}_{t+1}-\left(1+g_{t+1}\right) \widetilde{C F} t\right) \cdot\left(\widetilde{C F}_{s+1}-\left(1+g_{s}\right.\right.\right.$

Let $s<t$, then using the law of iterated expectation this can be rearranged to (see for example [13], p. 88)

$\operatorname{Cov}\left(\widetilde{\varepsilon}_{t+1}, \widetilde{\varepsilon}_{s+1}\right)=\mathrm{E}\left[\left(\widetilde{C F}{ }_{s+1}-\left(1+g_{s+1}\right) \widetilde{C F} s\right) \cdot \mathrm{E}\left[\widetilde{C F}_{t+1}-(1+\right.\right.$

But the right hand side is zero since

$\mathrm{E}\left[\left(\widetilde{C F}_{t+1}-\left(1+g_{t+1}\right) \widetilde{C F_{t}}\right) \mid \mathcal{F}_{s+1}\right]=\mathrm{E}\left[\mathrm{E}\left[\left(\widetilde{C F}_{t+1}-\left(1+g_{t+1}\right) \widetilde{C}\right.\right.\right.$ and therefore (8) is equivalent to uncorrelated increments.

We furthermore notice that the claimed structure of future cash flows (8) is the discrete time analogue of the assumption of a Brownian Motion. In the later case the stock price process satisfies

$$
\frac{d S}{S}=(r+d) d t+\sigma d W
$$

where $r$ is the drift of stock price, $d$ the (infinitesimal) dividend flow and $d W$ the corresponding Itô process. This is the same as to say that the (infinitesimal) increase $d S$ is uncorrelated to the current stock prize. Cash flow distributions as a Brownian Motion have been studied in a continuous time model by [1].

In fact relation (8) is not only necessary for the cost of capital and the ratio of cash flows to firm values to be deterministic but in the following sense sufficient.

Proposition 1 Assume the market is free of arbitrage. If two of the following conditions are satisfied the third follows:

(i) the cost of capital $\widetilde{k}_{t}$ are deterministic,

(ii) the cash flows are weak auto-regressive (i.e. there are positive real numbers $g_{t}$ such that relation (8) holds), (iii) the dividend ratios $\widetilde{d}_{t}$ are deterministic with $d_{t}>0$.

In the following we present some examples of cash flow processes with only one of the above assumptions and show that in this case in general the other two will not obtain. Consider the cost of capital as defined in (4):

$1+\widetilde{k}_{t+1}=\frac{\mathrm{E}\left[\widetilde{C F}_{t+1}+\widetilde{V}_{t+1} \mid \mathcal{F}_{t}\right]}{\widetilde{V}_{t}}=\frac{\mathrm{E}\left[\widetilde{C F}_{t+1}\left(1+\widetilde{d}_{t+1}^{-1}\right) \mid \mathcal{F}_{t}\right]}{\widetilde{V}_{t}}$

Assuming $\widetilde{d}_{t+1}$ deterministic this reads as

$$
1+\widetilde{k}_{t+1}=\left(1+d_{t+1}^{-1}\right) \widetilde{d}_{t}\left(1+\widetilde{g}_{t+1}\right),
$$

with $\widetilde{g}_{t+1}$ defined as

$$
\widetilde{g}_{t+1}=\frac{\mathrm{E}\left[\widetilde{C F}_{t+1} \mid \mathcal{F}_{t}\right]}{\widetilde{C F}_{t}}-1 .
$$

This is obviously only deterministic if both $\widetilde{g}_{t+1}$ and $\widetilde{d}_{t}$ are deterministic.

In our present formulation we assume that the distrib $\widetilde{a t j o n}$. of cash flows satisfy $\widetilde{C F} \neq 0$ in every period $t \leq T$. This rules out for instance the case of distributions having a single cash flow $\widetilde{C F}_{T}$ at time $T$ and no cash flows at any other time. In this case condition (8) would enforce $\mathrm{E}\left[\widetilde{C F}_{T}\right]=0$. In order to allow for zero cash flows at some periods, $\widetilde{C F}_{t}$ can be replaced by $\widetilde{V}_{t}$ in (8) leaving all results of the proposition valid.

Note that under the assumptions of proposition 1 the

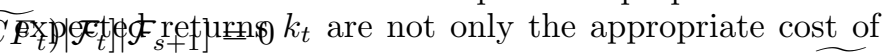
capital for the entire firm, but every single cash flow $\widetilde{C F}_{t}$ is to be valued using $k_{t}$ as discount rates. Hence, cost of capital turns out to be a simple way of evaluating the expected cash flows under the subjective probability measure. Cost of capital, which were defined as the expected return of the entire firm, are also the expected return of every single cash flow: 
Proposition 2 If the conditions of proposition 1 are satisfied for any $u>t \geq 1$, then the value of a single cash flow $\widetilde{C F}_{u}$ evaluated at time $t$ obtains as

$$
\frac{\mathrm{E}_{Q}\left[\widetilde{C F}_{u} \mid \mathcal{F}_{t}\right]}{\left(1+r_{f}\right)^{u-t}}=\frac{\mathrm{E}\left[\widetilde{C F}_{u} \mid \mathcal{F}_{t}\right]}{\prod_{s=t+1}^{u}\left(1+k_{s}\right)}
$$

Another consequence of our assumptions concerns the shape of the distribution of future cash flows. Projecting forward from time $t<u, \widetilde{C F}_{u}$ is given as

$\widetilde{C F}_{u}=\widetilde{C F}_{t}\left(1+\tilde{r}_{t+1}\right) \cdots\left(1+\tilde{r}_{u}\right) \frac{d_{t}^{-1} \cdots d_{u-1}^{-1}}{\left(1+d_{t+1}^{-1}\right) \cdots\left(1+d_{u}^{-1}\right)}$.

Therefore the shape of the cash flow distributions further ahead is principally given by the product of the one period return distributions $\tilde{r}_{t}$. Obviously with such a multiplicative structure certain regularities of the return distributions will lead to strong properties of the cash flow distributions. For instance, if the distribution of the returns are identical with a positive variance, then the pattern of projected distribution of the cash flows will have increasing variances and increasing skewness for longer investment horizons. This is a result that has been noticed by Fama in the case of a single cash flow realization in the last period. In Fama's analysis the multiplicative structure of the distributions is a consequence of the Bayesian expectation formation in a CAPM context. This holds true also in the present framework. The assumptions in proposition 1 make sure that no other effect masks this connection. In applied work many other interesting properties of the cash flow distributions can be deduced from the return distribution and vice versa with equation (11).

\section{Conclusion}

Capital budgeting of future uncertain cash flows with risk adjusted discount rates implies deterministic cost of capital. Beside, no further restriction on the shape or the evolution of the distribution of the cash flows is required when projects with many cash flow realizations are considered. Therefore our intuition that cash flows further ahead in the future should be more uncertain is wrong in general. Only when additional assumptions either on the price-dividend ratio of the investment or on the cash flow increments are made, further properties of the cash flow evolution can be deduced.

Clearly, in the real world we do observe increasing uncertainty for investments with longer investment horizons. Therefore a relationship between cash flows and returns similar to (11) is probably at hand. But this is not implying inevitably a specific behavior for the cash flow distributions in the future, since almost any pattern of future cash flow distributions can be constructed with (6). Evaluating future investment returns with the help of some asset pricing models helps to determine the distribution of one-period returns but does principally not restrict the shape of future cash flow distributions and the way the expectations thereof are formed. The optimistic message of this note is therefore, that we can safely rely on knowledge about the construction of investment returns without restricting our freedom to form expectations of future expected cash flows.

\section{Appendix}

\subsection{Proof of Proposition 1}

We start with $(i),(i i) \Longrightarrow(i i i)$. From (4), (8) and the law of iterated expectation it follows for all $t$

$$
\widetilde{V}_{t}=\widetilde{C F}_{t} \cdot \sum_{s=t+1}^{T} \frac{\left(1+g_{t+1}\right) \cdots\left(1+g_{s}\right)}{\left(1+k_{t+1}\right) \cdots\left(1+k_{s}\right)}=: \widetilde{C F}_{t} \cdot d_{t}^{-1}
$$

hence, the dividend ratios $d_{t}$ is deterministic.

Now $(i i),(i i i) \Longrightarrow(i)$. We have from (1), (4) and (for a proof of a similar result, see [4]):

$$
1+\widetilde{k}_{t}=\frac{\mathrm{E}\left[\left(1+d_{t}^{-1}\right) \widetilde{C F}_{t} \mid \mathcal{F}_{t-1}\right]}{d_{t-1}^{-1} \widetilde{C F}_{t-1}}=\left(1+d_{t}^{-1}\right)\left(1+g_{t}\right) d_{t-1}
$$

and hence the cost of capital must be deterministic.

To show $(i),(i i i) \Longrightarrow(i i)$ we start with

$$
\begin{aligned}
\left(1+k_{t}\right) V_{t-1} & =\mathrm{E}\left[\widetilde{C F}_{t}+\widetilde{V}_{t} \mid \mathcal{F}_{t-1}\right] \\
\frac{1+k_{t}}{1+d_{t}^{-1}} d_{t-1} \widetilde{C F}_{t-1} & =\mathrm{E}\left[\widetilde{C F}_{t} \mid \mathcal{F}_{t-1}\right]
\end{aligned}
$$

and this is indeed (8).

\subsection{Proof of Proposition 2}

We show the claim for $s=t-1$. Since the dividend ratio is deterministic we have

$$
\begin{aligned}
\frac{\mathrm{E}_{Q}\left[d_{t}^{-1} \widetilde{C F}_{t}+\widetilde{C F}_{t} \mid \mathcal{F}_{t-1}\right]}{1+r_{f}} & =\widetilde{V}_{t-1}=\frac{\mathrm{E}\left[d_{t}^{-1} \widetilde{C F}_{t}+\widetilde{C F}_{t} \mid \mathcal{F}_{t-1}\right]}{1+k_{t}} \\
\Longrightarrow \quad \frac{\mathrm{E}_{Q}\left[\widetilde{C F}_{t} \mid \mathcal{F}_{t-1}\right]}{1+r_{f}} & =\frac{\mathrm{E}\left[\widetilde{C F}_{t} \mid \mathcal{F}_{t-1}\right]}{1+k_{t}}
\end{aligned}
$$

which is the claim for $s=t-1$.

By taking the expectation $\mathrm{E}_{Q}\left[\cdot \mid \mathcal{F}_{t-2}\right]$ and using (8) in the expression on the right hand side of equation (12) we find

$\frac{\mathrm{E}_{Q}\left[\widetilde{C F}_{t} \mid \mathcal{F}_{t-2}\right]}{1+r_{f}}=\frac{\mathrm{E}_{Q}\left[\mathrm{E}\left[\widetilde{C F}_{t} \mid \mathcal{F}_{t-1}\right] \mid \mathcal{F}_{t-2}\right]}{1+k_{t}}=\frac{\mathrm{E}_{Q}\left[\left(1+g_{t}\right) \widetilde{C F}_{t-1} \mid \mathcal{F}_{t-2}\right]}{1+k_{t}}$

Using (12) (with $t-1$ instead of $t$ ) the right hand side simplifies to

$$
\frac{\mathrm{E}_{Q}\left[\widetilde{C F}_{t} \mid \mathcal{F}_{t-2}\right]}{1+r_{f}}=\frac{\left(1+g_{t}\right) \frac{\left(1+r_{f}\right) \mathrm{E}\left[\widetilde{C F}_{t-1} \mid \mathcal{F}_{t-2}\right]}{1+k_{t-1}}}{1+k_{t}}
$$


or again with (8)

$$
\frac{\mathrm{E}_{Q}\left[\widetilde{C F}_{t} \mid \mathcal{F}_{t-2}\right]}{\left(1+r_{f}\right)^{2}}=\frac{\mathrm{E}\left[\widetilde{C F}_{t} \mid \mathcal{F}_{t-2}\right]}{\left(1+k_{t}\right)\left(1+k_{t-1}\right)}
$$

This is the claim for $s=t-2$. Continuing our calculations we arrive at the desired result.

\section{References}

1. Ang A, Liu J (2004) Risk, return, and dividends, Discussion Paper, UCLA.

2. Back K, Pliska SR (1991) On the fundamental theorem of asset pricing with an infinite state space, Journal of Mathematical Economics 20: 1-18.

3. Black F (1988) A simple discounting rule, Financial Management 17(2): 7-11.

4. Canefield D (1999) Some remarks on the valuation of firms, Journal of Valuation 4: 23-25.

5. Fama EF (1977) Risk-adjusted discount rates and capital budgeting under uncertainty, Journal of Financial Economics 5: 3-24.

6. Fama EF (1996) Discounting under uncertainty, Journal of Business 69 (4): 415-428.

7. Franke G (1984) Conditions for myopic valuation and serial independence of the market excess return in discrete time models, Journal of Finance 39 (2): 425-442.

8. Geske R (1978) The pricing of options with stochastic dividend yield, Journal of Finance 33: 617-625.

9. Merton RC (1974) On the pricing of corporate debt, the risk structure of interest rates, Journal of Finance 29: 449470.

10. Myers SC, Turnbull SM (1977) Capital budgeting and the capital asset pricing model, good news and bad news, Journal of Finance 32: 321-333.

11. Ohlson JA (1995) Earnings, book values, and dividends in equity valuation, Contemporary Accounting Research 11: 661-687.

12. Sick GA (1986) A certainty-equivalent approach to capital budgeting, Financial Management 15: 23-32.

13. Williams D (1991) Probability with Martingales Cambridge University Press, Cambridge. 